\title{
TRPC1 channels regulate directionality of migrating cells
}

\author{
A. Fabian • T. Fortmann $\cdot$ P. Dieterich $\cdot$ \\ C. Riethmüller • P. Schön • S. Mally • B. Nilius • \\ A. Schwab
}

Received: 18 December 2007 /Revised: 10 March 2008 / Accepted: 7 April 2008 / Published online: 30 April 2008

(C) Springer-Verlag 2008

\begin{abstract}
Cell migration depends on the generation of structural asymmetry and on different steps: protrusion and adhesion at the front and traction and detachment at the rear part of the cell. The activity of $\mathrm{Ca}^{2+}$ channels coordinate these steps by arranging intracellular $\mathrm{Ca}^{2+}$ signals along the axis of movement. Here, we investigated the role of the putative mechanosensitive canonical transient receptor potential channel 1 (TRPC1) in cell migration. We analyzed its function in transformed renal epithelial (Madin-Darby canine kidney-focus) cells with variation of TRPC1 expression. As shown by time lapse video microscopy, TRPC1 knockdown cells have partially lost their polarity and the ability to persistently migrate into a given direction. This failure is linked to the suppression of a local $\mathrm{Ca}^{2+}$ gradient at the front of migrating TRPC1 knockdown cells,
\end{abstract}

Electronic supplementary material The online version of this article (doi:10.1007/s00424-008-0515-4) contains supplementary material, which is available to authorized users.

A. Fabian $(\triangle) \cdot$ T. Fortmann · C. Riethmüller $\cdot$ P. Schön •

S. Mally $\cdot$ A. Schwab

Institute of Physiology II, University of Münster,

Robert-Koch-Str. 27b,

48149 Münster, Germany

e-mail: afabian@uni-muenster.de

P. Dieterich

Institute of Physiology, TU,

Dresden, Germany

P. Schön

Life Science Team, Veeco Instruments GmbH,

Mannheim, Germany

B. Nilius

Department of Molecular Cell Biology,

Lab Ion Channel Research, Campus Gasthuisberg, KU,

Leuven, Belgium whereas TRPC1 overexpression leads to steeper $\mathrm{Ca}^{2+}$ gradients. We propose that the $\mathrm{Ca}^{2+}$ signaling events regulated by TRPC1 within the lamellipodium determine polarity and directed cell migration.

Keywords TRPC1 $\cdot$ Cell migration · Polarity

Calcium signaling

\section{Introduction}

Cell migration is an essential physiological process within the human body. Even after embryonic development, there are a number of cells that continuously migrate through the body. Among these are immune cells which migrate to sites of inflammation or stem cells and fibroblasts which warrant the maintenance of diverse tissues. In addition, migration of cancer cells is necessary for tumor invasion and metastasis and therefore represents a critical step in the course of the disease.

A basic principle of cell migration is the functional and morphological polarity of migrating cells. Motile cells protrude a lamellipodium at the front, where new focal adhesions are formed and traction force is exerted. This action gives rise to progressive stretching of the cell body; the detachment of the rear adhesions completes the migration process $[34,38]$. For sustained movement, forward cell protrusion has to be coordinated with rear cell retraction. At this point, $\mathrm{Ca}^{2+}$ channels may come into play because they are able to integrate signals from the front and the rear part of migrating cells into an increase of the intracellular second messenger $\mathrm{Ca}^{2+}$. Many factors involved in cell migration are regulated by $\mathrm{Ca}^{2+}$ including myosin light chain kinase, calpain, gelsolin, calcineurin, and 
integrins $[1,11,12,23]$. In fact, repetitive $\mathrm{Ca}^{2+}$ transients or cytoplasmic $\mathrm{Ca}^{2+}$ gradients were shown in migrating neurons, neutrophils, fibroblasts, eosinophils, tumor cells, and other cell types $[5,16,18,22,26]$. The precise temporal and spatial regulation of $\left[\mathrm{Ca}^{2+}\right]_{i}$ is required for cellular motility. As a consequence, $\mathrm{Ca}^{2+}$-permeable channels, the plasma membrane $\mathrm{Ca}^{2+}$-adenosine triphosphatase, and especially the $\mathrm{Na}^{+}-\mathrm{Ca}^{2+}$ exchanger are decisive for cell migration [10]. Moreover, $\left[\mathrm{Ca}^{2+}\right]_{\mathrm{i}}$ gradients have been shown to be important in determining cellular polarity and thereby directionality $[16,20,36]$.

$\mathrm{Ca}^{2+}$ transients can be evoked by the action of $\mathrm{Ca}^{2+}$ permeable stretch-activated channels which appear to be gated by membrane stretch that, in migrating cells, could be provoked by the protruding lamellipodium or directly by tension generated by actomyosin forces at sites of adhesion to the matrix [9, 17]. Local increases in traction force during the movement of fish keratocytes [8, 9, 24] and fibroblasts [28] have been connected to such $\mathrm{Ca}^{2+}$ transients. The nonspecific blocker of stretch-activated cation channels gadolinium was used to block $\mathrm{Ca}^{2+}$ transients and impaired migration in these studies. Although all these publications stressed the importance of the function of $\mathrm{Ca}^{2+}$ in cell migration, the molecular identity of $\mathrm{Ca}^{2+}$ channels involved in this process is still unknown. Interesting candidates are members of the transient receptor potential (TRP) family of cation channels. We have recently shown that activation of the TRP vanilloid 1 channel (TRPVA) accelerates migration of human hepatoblastoma cells [44]. Here, we study the effect of TRP cation channel subfamily $\mathrm{C}$ member 1 (TRPC1) on migration of transformed renal epithelial (Madin-Darby canine kidney-focus (MDCK-F)) cells. TRPC1 is a $\mathrm{Ca}^{2+}$-permeable TRP channel $[45,46]$ that could be involved in the regulation of cell migration. This channel has been proposed to form a stretch-activated cation channel or to be at least a subunit of such a channel, although the functional expression of TRPC1 as mechanosensing channel has been challenged $[14,27,30]$. A detailed review critically reevaluates the role of TRP channels as mechanosensitive channels [39]. Nonetheless, a function of TRPC1 has been shown in the migration of intestinal epithelial cells during closure of a wounded epithelial layer [32]. In the present study, we investigated the role of the putative mechanosensitive $\mathrm{Ca}^{2+}$ channel TRPC1 in single-cell migration of MDCK-F cells.

\section{Materials and methods}

Cells and cell culture

Experiments were carried out on transformed MDCK-F cells [29] grown in bicarbonate-buffered minimal essential medium (MEM; pH 7.4) with Earle's salts (PAA Laboratories, Austria) supplemented with $10 \%(v / v)$ fetal calf serum (Biochrom, Germany) at $37^{\circ} \mathrm{C}$ in a humidified atmosphere of $5 \% \mathrm{CO}_{2}$.

Transfections were carried out with FuGENE 6 (Roche, Germany), according to the manufacturers' protocols. The pcDNA3 plasmid containing the human TRPC1 had an Nterminal insertion of the hemagglutinin (HA) tag. We generated a polyclonal cell line, stably overexpressing this construct, termed hTRPC1-HA. Additionally, we transfected MDCK-F cells with the pcDNA3 plasmid alone, which served as control. The selection of stably transfected cells was performed with medium containing $0.6 \mathrm{~g} / \mathrm{L}$ geneticin (G-418-sulfate; PAA Laboratories, Austria). The siRNA-treated cells were selected in medium containing $2.5 \mathrm{mg} / \mathrm{L}$ puromycin (PAA Laboratories, Austria).

\section{Silencing of TRPC1 expression by small interfering RNA}

Oligonucleotides harboring the respective 19-nt target sequences were ligated into the pSUPER.retro.puro vector (OligoEngine, USA) following the published method [4]. Vectors were verified by sequencing. For full oligonucleotide and targeting sequences, see supplementary information. Cells were transfected overnight with the respective vectors and then replated into selection medium containing puromycin and several cell clones were obtained by single-cell dilution. We used two clones with reduced TRPC1 expression, referred to as SITRPCl cells. The efficiency of gene product suppression was revealed by reverse-transcription polymerase chain reaction (RT-PCR) as described below. Mock transfections were performed with pSUPER.retro.puro harboring a mock sequence followed by puromycin selection. Via single-cell dilution, we isolated the mock control clone.

Analysis of cell migration

Migration of MDCK-F cells was monitored by means of time lapse microscopy as described [37]. Cells were seeded on tissue culture flasks coated with $1 \mu \mathrm{g} / \mathrm{cm}^{2}$ fibronectin (Roche, Germany) 1day prior to experiments. The culture flasks with the cells were put into heated chambers on stages of inverted microscopes (Axiovert25, Carl Zeiss, Inc., Germany). Cell migration was recorded in 1-min intervals for $3 \mathrm{~h}$ at $37^{\circ} \mathrm{C}$ using video cameras (Models XCST70CE and XC-77CE, Hamamatsu-Sony, Japan) and PCvision frame grabber boards (Hamamatsu, Herrsching, Germany). Acquisition of images was controlled by HiPic and WASABI softwares (Hamamatsu). The outlines of the cells were marked at each time step throughout the entire image stack applying the AMIRA software (Mercury Computer Systems, USA). The cell contours then served 
as the basis for further analysis. Parameters such as structural index, migratory velocity (micrometer per minute) and translocation (micrometer) were analyzed using self-made JAVA programs and the National Institutes of Health ImageJ software (http://rsb.info.nih.gov/ij/). Migration was determined as the movement of the cell center as a function of time. We calculated the speed (micrometer per minute) by applying a three-point difference quotient and the displacement (micrometer), which is the distance covered by a cell during a given time period. The combined trajectories of a cell population allow the calculation of the mean squared displacement (msd). The msd (micrometer ${ }^{2}$ ) describes the mean of the squared distances between a common starting point at time $t_{0}$ and the actual positions of a cell population at time $t$,

$\operatorname{msd}(t)=\left\langle\left[x\left(t+t_{0}\right)-x\left(t_{0}\right)\right]^{2}+\left[y\left(t+t_{0}\right)-y\left(t_{0}\right)\right]^{2}\right\rangle$.

To analyze directionality of migration (persistence), we quantified the angle at which protrusions occurred with respect to the trajectory of the cell. Cells with one lamellipodium and a high degree of directionality move with angles centering around $\pm 0^{\circ}$. Angles greater than $\pm 100^{\circ}$ indicate a low degree of directionality due to the coexistence of several lamellipodia or due to a complete change in the direction of movement.

Moreover, we used the time lapse movies to investigate the morphology of migrating cells. The cell area was measured as the sum of all pixels inside the cells' contours. The structural index is a shape factor which relates cell area $(A)$ to its perimeter $(P)$,

Structural index $=4 \pi A / P^{2}$.

Values close to "1" correspond to a spherical and values close to " 0 " represent elongated cells with several protrusions.

In paired experiments, we tested whether elevating $\left[\mathrm{Ca}^{2+}\right]_{\mathrm{i}}$ in siTRPC1 cells has any effect on cell migration: after a control period (30min), we applied low doses of the $\mathrm{Ca}^{2+}$ ionophore ionomycin $(50 \mathrm{nmol} / \mathrm{L}$; MP Biomedicals, France) and monitored migration for additional $30 \mathrm{~min}$. To inhibit mechanosensitive cation channels, we employed a peptide (GsMTx-4, Peptides International, USA) isolated from venom of the tarantula, Grammostola spatulata $[3,40$, 41]. The effect of GsMTx-4 was studied in paired experiments. A 30-min control period was followed by an experimental period (at least $180 \mathrm{~min}$ ) in the presence of $10 \mu \mathrm{mol} / \mathrm{L}$ GsMTx-4.

Intracellular $\mathrm{Ca}^{2+}$ measurements

One day prior to the experiment, cells were seeded on fibronectin-coated $\left(1 \mu \mathrm{g} / \mathrm{cm}^{2}\right)$ glass coverslips. The cells were preincubated with 4-(2-hydroxyethyl)-1-piperazineethanesulfonic acid (HEPES)-buffered MEM for 10min and subsequently fura-2-AM ( $3 \mu \mathrm{mol} / \mathrm{L}$; Calbiochem) [15] was added and for dye loading at room temperature for $20 \mathrm{~min}$. The experiments were carried out at $37^{\circ} \mathrm{C}$, and cells were continuously superfused with Ringer solution $(\mathrm{NaCl}$ $122.5 \mathrm{mmol} / \mathrm{L}, \mathrm{KCl} 5.4 \mathrm{mmol} / \mathrm{L}, \mathrm{CaCl}_{2} 1.2 \mathrm{mmol} / \mathrm{L}, \mathrm{MgCl}_{2}$ $0.8 \mathrm{mmol} / \mathrm{L}$, D-glucose $5.5 \mathrm{mmol} / \mathrm{L}$, HEPES $10.0 \mathrm{mmol} / \mathrm{L}, \mathrm{pH}$ 7.4). Excitation wavelength alternated between 340 and $380 \mathrm{~nm}$. The emitted fluorescence was monitored at $500 \mathrm{~nm}$. CoolSnap camera, monochromator, and data acquisition were controlled by the Metafluor Software (Visitron Systems). Fluorescence intensities were corrected by background subtraction and measured in 10-s intervals in three distinct regions from front to back of migrating cells: leading edge $(0-5 \mu \mathrm{m})$, lamella $(5-10 \mu \mathrm{m})$, and rear (final $5 \mu \mathrm{m}$ of the cell). $\left[\mathrm{Ca}^{2+}\right]_{\mathrm{i}}$ was calculated as described before [10]. Briefly, $\mathrm{Ca}^{2+}$ measurements were calibrated at the end of each experiment. Maximal and minimal ratios were determined separately for each region by the application of ionomycincontaining $(1 \mu \mathrm{mol} / \mathrm{L})$ Ringer solutions containing either $5 \mathrm{mmol} / \mathrm{L} \mathrm{Ca}^{2+}$ or $5 \mathrm{mmol} / \mathrm{L}$ ethylene glycol tetraacetic acid. For each cell clone, at least 29 cells were analyzed and mean values and SEM of the ratios are presented.

\section{Adhesion assay}

To analyze the adhesiveness of MDCK-F cells, we allowed 30,000 cells per well to adhere to fibronectincoated $\left(1 \mu \mathrm{g} / \mathrm{cm}^{2}\right)$ 24-well plates in serum-containing culture medium. After 30 -min incubation at $37^{\circ} \mathrm{C}$ in a $\mathrm{CO}_{2}$ atmosphere, weakly adherent cells were removed by washing with phosphate-buffered saline. Adherent cells were fixed and counted.

\section{RT-PCR}

Total RNA from MDCK-F cells grown to confluence was isolated with RNAeasy spin columns (Qiagen, Germany) 1 day after plating. Same amounts of each RNA sample were used for cDNA synthesis with SuperScript III reverse transcriptase (Invitrogen, Germany) using oligo (dT) ${ }_{16}$ primers according to the manufacturers' protocols. TRPC1 transcripts were either amplified with primers specific for canine and human TRPC1 isoforms to show the knockdown of endogenous TRPC1 or the overexpression of the human HA-tagged TRPC1 isoform in MDCK-F cells. The amounts of TRPC1 mRNA were normalized with $\beta$-actin mRNA. The annealing temperature was $53^{\circ} \mathrm{C}$ and 30 cycles were performed in an Eppendorff Mastercycler (Eppendorff, Germany). Amplified cDNA fragments were size-fractionated in a $1 \%$ agarose gel and stained with SYBR Gold (Molecular Probes, USA). 
Cell volume measurements with atomic force microscopy

Cell imaging was performed in standard contact mode as described [35]. Briefly, atomic force microscopy (AFM) measurements of glutaraldehyde-fixed MDCK-F cells on fibronectin-coated cell culture dishes were performed in HEPES-buffered solution ( $\mathrm{pH}$ 7.4) using a Bioscope II controlled by Nanoscope 7.20 software (VEECO, Mannheim, Germany). Tips with a radius of $8 \mathrm{~nm}$ on rectangular goldcoated silicon nitride cantilevers were used $(0.09 \mathrm{~N} / \mathrm{m}$, Olympus Biolever mini BL-AC40TS-C2, purchased through LOT-Oriel. Darmstadt, Germany). The volume of a cell was enumerated by the "bearing" option in software Nanoscope $5.48 \mathrm{~b} 12$ after linear "plane fit" of the datasets.

\section{Statistics}

All experiments were repeated three to six times. Data are presented as the mean values \pm SEM. The data were tested for significance employing Student's unpaired $t$ test or paired $t$ test where applicable. The level of significance was set at $p<0.05$.

\section{Results}

TRPC1 expression level correlates with efficient movement of MDCK-F cells

To investigate the function of the TRPC1 channel in cell migration, we altered the expression levels of endogenously expressed TRPC1 in MDCK-F cells. We reduced TRPC1 expression by means of siRNA technology. We expressed siRNA with the $p S U P E R$ vector system and generated two siTRPC1 clones. The stable knockdown to $30 \%$ and $70 \%$ (clone $5 \mathrm{~A}$ and $6 \mathrm{D}$, respectively) of the expression in the respective control cells was determined by RT-PCR (Fig. 1a). The amplification of TRPC1 transcripts was normalized using $\beta$-actin probes. Moreover, we generated a polyclonal MDCK-F cell line stably overexpressing the human TRPC1-HA. The overexpression of TRPC1 was quantified in the same way. The increase of TRPC1 expression is approximately twofold (Fig. 1b). The following data refer mostly to the siTRPC1 clone $5 \mathrm{~A}$ with knockdown to $30 \%$ of normal TRPC1 expression.

Silencing of TRPC1 clearly changes the morphology of MDCK-F cells as shown in video 1 (see supplementary material) and in the image series taken from this movie displayed in Fig. 2. siTRPC1 cells form multiple active lamellipodia often coexisting at opposing sides of the cell. In contrast, the respective control (not shown) as well as hTRPC1-HA-overexpressing cells are clearly polarized and migrate with one leading lamellipodium. In line with this,

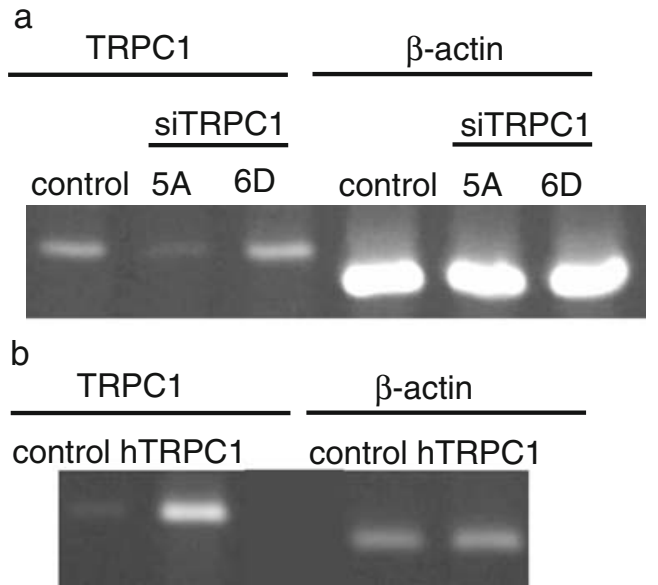

Fig. 1 TRPC1 expression is varied by knockdown and overexpression of TRPC1. a RT-PCR of siTRPC1 and mock control cells. Compared to mock transfected controls TRPC1 expression was reduced to $30 \%$ and $70 \%$ in siTRPC1 cell clones $5 \mathrm{~A}$ and $6 \mathrm{D}$, respectively. b RT-PCR of MDCK-F cells transfected with an "empty" vector and the human-TRPC1-HA construct, "control," and "hTRPC1," respectively. The mRNA level of human TRPC1-HA has at least doubled when compared with endogenous TRPC1 mRNA levels in MDCK-F cells. Amplification of TRPC1-transcripts in RTPCR was normalized to $\beta$-actin mRNA

the structural index (area-perimeter relation) of knockdown cells is much lower than that of hTRPC1-HA-overexpressing cells $(0.21 \pm 0.02$ with $n=33$ and $0.41 \pm 0.03$ with $n=26$, respectively). Besides the changed morphology, the mode of migration of siTRPC1 cells has also visibly changed. The speed of migration of siTRPC1 cells is significantly lower than that of hTRPC1-HA-overexpressing cells: $0.88 \pm$ $0.03 \mu \mathrm{m} / \mathrm{min}(n=33)$ versus $1.06 \pm 0.04 \mu \mathrm{m} / \mathrm{min}(n=26)$. This is paralleled by a pronounced decrease of the displacement (distance between start and end position at $t=$ 180min). siTRPC1 cells reach a mean distance of $68.7 \pm$ $5.5 \mu \mathrm{m}(n=33)$, whereas MDCK-F cells overexpressing hTRPC1-HA cover $89.2 \pm 7.7 \mu \mathrm{m}$ within $180 \mathrm{~min}(n=26)$. Figure $3 \mathrm{a}$ shows the mean displacements (radii of circles) and the trajectories. Both siTRPC1 clones, independently of their knockdown efficiency, have a strongly decreased msd, opposing to hTRPC1-HA-overexpressing cells which show an increase of msd compared to vector and mock control lines (Fig. 3c). The slopes of the msd curves allow the classification of the movement. The suppression of TRPC1 converts the normally super diffusive mode of migration of MDCK-F cells into a diffusive or random movement [7].

Closer inspection of the trajectories reveals a "noisy" movement of siTRPC1 cells pointing to frequent changes in the direction of movement (Fig. 3b). We therefore analyzed the angles at which cell protrusions occur with respect to the cells' trajectories. Cells with one prominent lamellipodium and a high level of directionality (persis- 


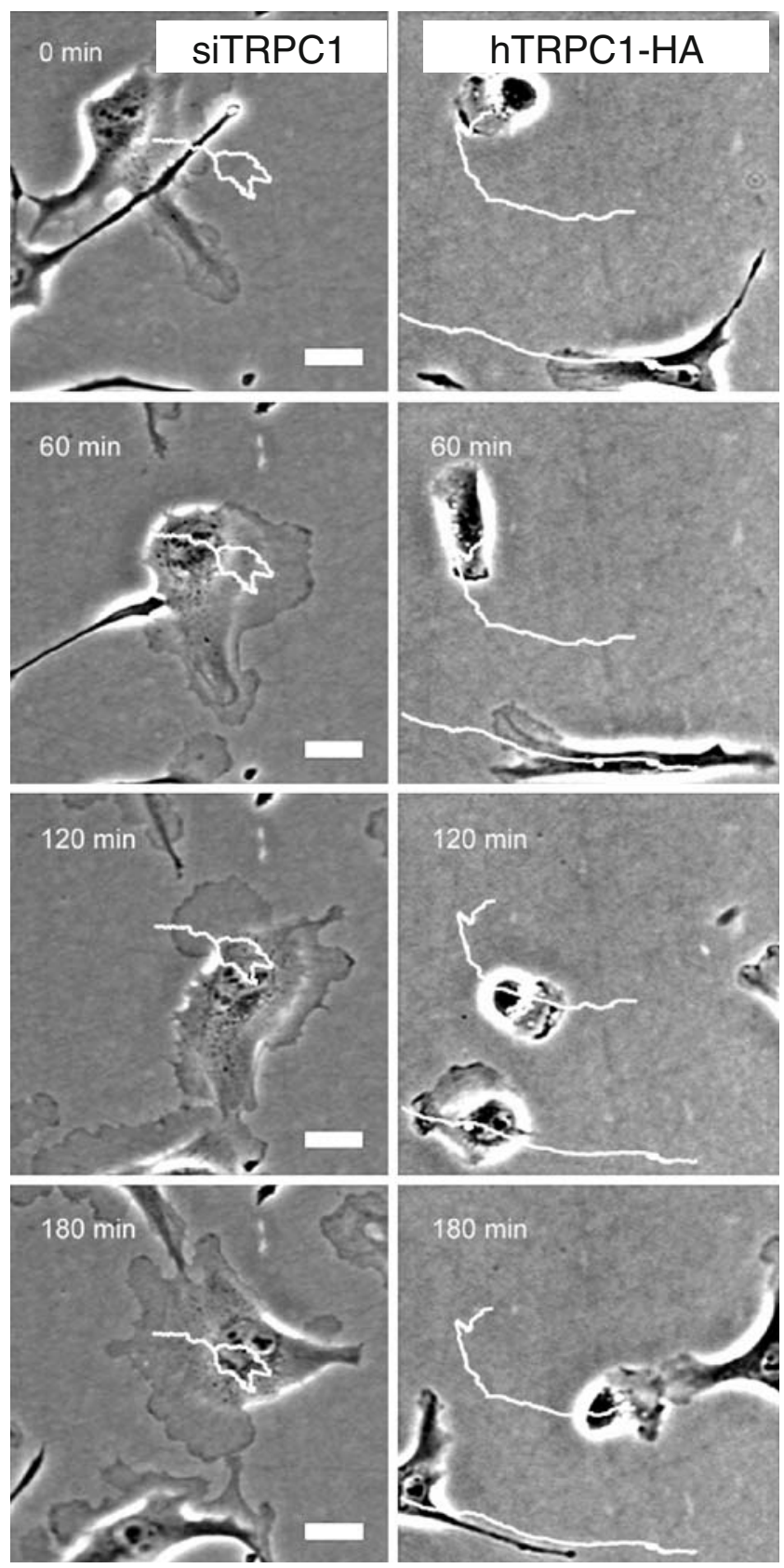

Fig. 2 TRPC1-depleted cells have a polarization defect and cell migration is impaired. Images of time lapse movies with 60 -min intervals show that siTRPC1 cells (left panel) are not able to polarize anymore and instead form multiple protrusions into several directions simultaneously. Moreover, TRPC1-depleted cells do not translocate efficiently as shown by the superimposed trajectory of the cell. In contrast, hTRPC1-HA-overexpressing MDCK-F cells (right panel) are clearly polarized and move over greater distances in a wellcoordinated way. Scale bar $25 \mu \mathrm{m}$

tence) generate angles centering around $\pm 0^{\circ}$. Angles around $\pm 180^{\circ}$ indicate that the cells reverse the direction of movement with protrusions occurring at the rear part. The normalized histograms of angles from siTRPC1, from mock control, and from hTRPC1-HA-transfected cells are shown in Fig. 4. Mock controls as well as hTRPC1-HA-overexpressing cells display a high degree of persistence as indicated by the peak of the distribution at an angle of $\sim 0^{\circ}$ ( $n=24$ and $n=26$ ). Silencing of TRPC1 expression results in a flattening of the distribution that implies a much higher incidence of protrusions occurring at angles of greater than

a

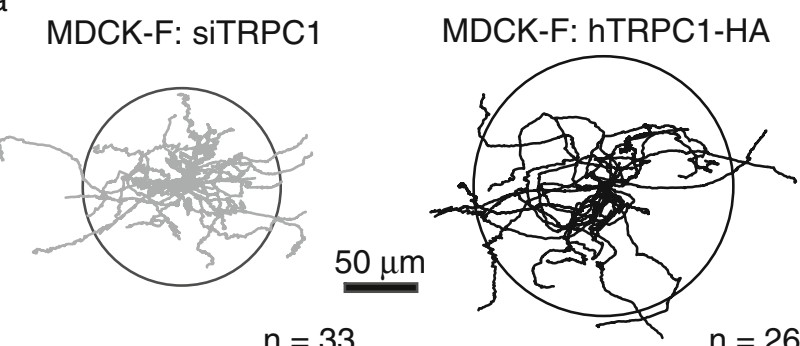

b

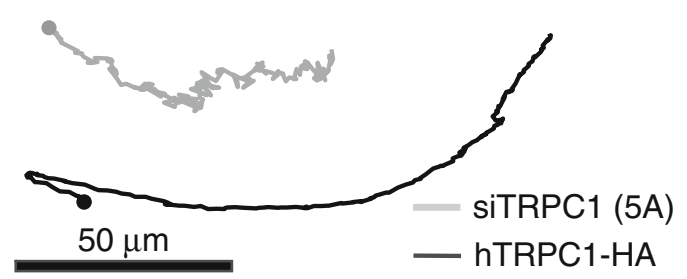

C

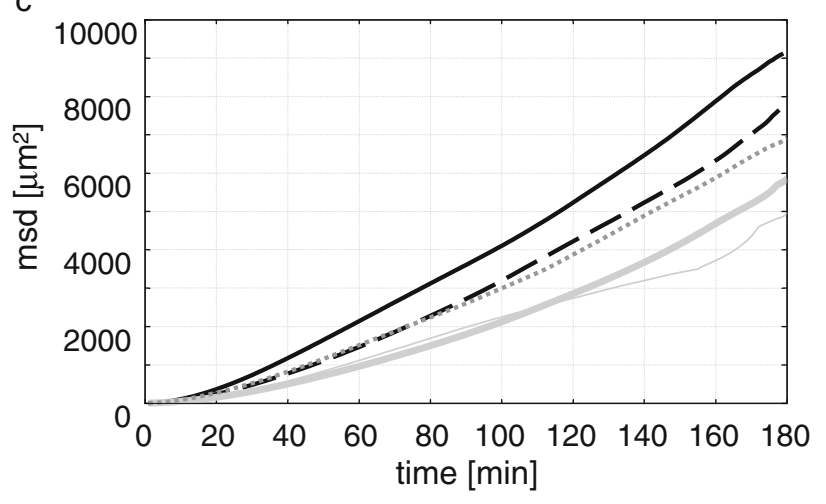

Fig. 3 The translocation of siTRPC1 cells is significantly reduced. a Trajectories were normalized to a common starting point. The circles indicate the mean distance covered within $180 \mathrm{~min}$. Silencing of TRPC1 expression leads to shorter migration paths with lower directionality in comparison to hTRPC1-HA overexpression. Scale bar $50 \mu \mathrm{m}$. b Magnification of a representative trajectory of a siTRPC1 cell (gray) shows the "noisy" movement, whereas a hTRPC1-HA-overexpressing cell shows nearly no turning. Starting points are indicated by dots. $\mathbf{c}$ The mean squared displacement $(\mathrm{msd})$ of TRPC1 knockdown cells (clone 5A: bold gray, clone 6D: gray) is strongly decreased, whereas msd of cells with overexpression of hTRPC1-HA (black) is significantly increased when compared to the respective control cells - mock control cells (dotted gray) or pcDNA3 vector control (dashed black) 


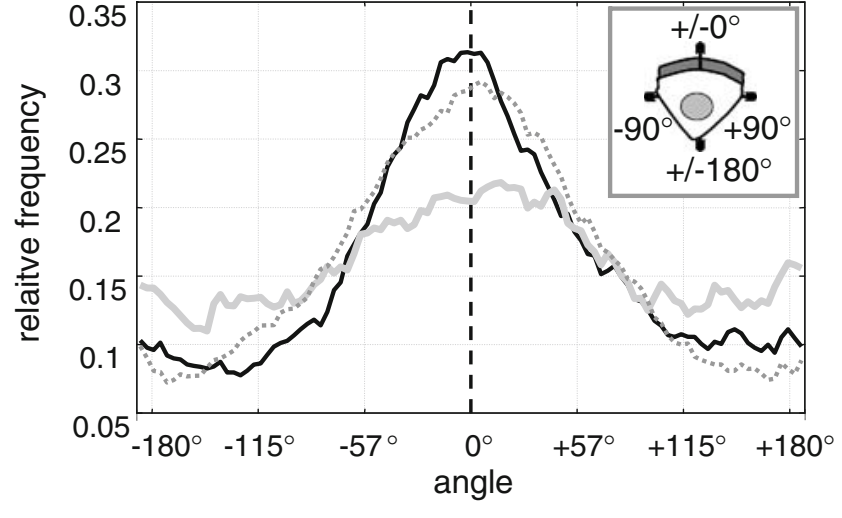

- hTRPC1-HA mock control $=$ siTRPC1 (5A)

Fig. 4 TRPC1 ablation leads to partial loss of cellular directionality. The distribution of angles at which protrusions occur with respect to the migration paths represents the degree of directionality of cellular movement (persistence). Cells that overexpress hTRPC1-HA (black) as well as mock cells (dotted gray) show a high degree of persistence as shown by the peak of the distribution at an angle of $\sim 0^{\circ}$. In contrast, siTRPC1 cells (gray) have mainly a random orientation with angles more than $\pm 100^{\circ}$ indicating the frequent occurrence of uncoordinated protrusions

$\pm 100^{\circ}(n=33)$. Cells with a less efficient knockdown of TRPC1 expression (clone 6D: not shown) have also an increased tendency of $180^{\circ}$ turning of their migration direction. These findings provide strong evidence that TRPC1 is involved in the maintenance of cellular polarity in migrating cells and mediates directionality.

Blockade of mechanosensitive cation channels impairs cell migration

The peptide GsMTx-4 isolated from the venom of the tarantula G. spatulata, an inhibitor of mechanosensitive cation channels [3, 40, 41], was employed to verify the function of such channels in cell migration (Fig. 5). As already shown, the displacement at $t=30 \mathrm{~min}$ of siTRPC1 cells $(13.4 \pm 1.7 \mu \mathrm{m}, n=29)$ is much lower than that of cells overexpressing hTRPC1-HA $(23.7 \pm 2.0 \mu \mathrm{m}, n=33)$. However, GsMTx-4 reduces the displacement of both cell lines to the same low basal level. The respective values for siTRPC1 and hTRPC1-HA cells are $8.4 \pm 1.3$ and $9.8 \pm$ $1.0 \mu \mathrm{m}$, respectively. Started differently, GsMTx-4 is three times more effective in cells overexpressing TRPC1-HA than in cells with reduced expression. These findings provide indirect evidence that GsMTx-4 may not only block TRPC6 [40] but also TRPC1. Another hint into this direction comes from measurements of resting $\mathrm{Ca}^{2+}$ before and immediately after $(3 \mathrm{~min})$ application of $10 \mu \mathrm{mol} / \mathrm{L}$ GsMTx-4. hTRPC1-HA cells show a significant decrease of resting $\mathrm{Ca}^{2+}$ after treatment with the venom, whereas siTRPC1 cells display no difference (data not shown). Alternatively, the differential response of both cell types to
GsMTx- 4 could reflect the fact that TRPC1 is an essential component of a heteromultimeric channel that is sensitive to this toxin.

TRPC1 generates a lamellipodial $\mathrm{Ca}^{2+}$ gradient

To obtain deeper insights into the mechanism underlying the defects in migration of TRPC1 knockdown cells, we measured $\left[\mathrm{Ca}^{2+}\right]_{\mathrm{i}}$ ratiometrically in three distinct areas of migrating cells: the leading edge, the consecutive lamella, and the rear part. The first two areas correspond to distinct arrays of F-actin that determine the protrusive activity of migrating cells [31]. The results of these experiments are shown in Fig. 6a. We found that $\left[\mathrm{Ca}^{2+}\right]_{\mathrm{i}}$ is higher at the leading edge of hTRPC1-HA cells and mock control cells $(141 \pm 29 \mathrm{nmol} / \mathrm{L}, n=29$ and $108 \pm 12 \mathrm{nmol} / \mathrm{L}, n=40$, respectively) than in the lamella $(118 \pm 24$ and $104 \pm$ $12 \mathrm{nmol} / \mathrm{L}$, respectively). Overexpression of TRPC1 amplifies this gradient. In contrast, the $\mathrm{Ca}^{2+}$ gradient between leading edge and lamella is absent in siTRPC1 cells. Moreover, the mean $\left[\mathrm{Ca}^{2+}\right]_{\mathrm{i}}$ of TRPC1 knockdown cells is strongly reduced - leading edge $63 \pm 5 \mathrm{nmol} / \mathrm{L}$ and lamella $62 \pm 5 \mathrm{nmol} / \mathrm{L}(n=64)$. Figure $6 \mathrm{~b}$ summarizes our findings: $86 \%$ of hTRPC1-HA-overexpressing cells and $64 \%$ of mock control cells have a gradient with higher local $\left[\mathrm{Ca}^{2+}\right]_{\mathrm{i}}$ in the leading edge than in the lamella but only $45 \%$ of siTRPC1 cells. We can conclude that the lamellipodial gradient of $\left[\mathrm{Ca}^{2+}\right]_{\mathrm{i}}$ is directly correlated with TRPC1 expression.

Because mean $\left[\mathrm{Ca}^{2+}\right]_{\mathrm{i}}$ independent of a $\mathrm{Ca}^{2+}$ gradient may also affect cell migration of siTRPC1 cells, we increased $\left[\mathrm{Ca}^{2+}\right]_{\mathrm{i}}$ artificially by adding a low dose of the $\mathrm{Ca}^{2+}$ ionophore ionomycin $(50 \mathrm{nmol} / \mathrm{L})$ to the medium.

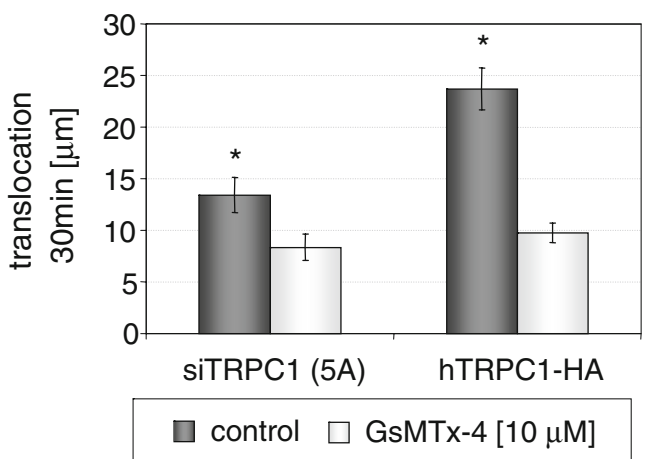

Fig. 5 Blockade of TRPC1 with GsMTx-4 strongly impairs cell migration. The peptide GsMTx-4 is the venom of a tarantula and was used in paired migration experiments to inhibit stretch-activated cation channels. The mean displacement of siTRPC1 cells at $t=30 \mathrm{~min}$ is much lower than that of cells overexpressing hTRPC1-HA. GsMTx-4 is three times more effective in cells overexpressing TRPC1-HA than in cells with reduced expression because the displacement upon application of the drug is decreased to the same basal level in both cell types. Asterisks, $p<0.05$ 
Ionomycin has no impact on $\mathrm{Ca}^{2+}$ gradient formation, but it elevates the average $\left[\mathrm{Ca}^{2+}\right]_{\mathrm{i}}$ in siTRPC1 cells by $41 \pm$ $13 \mathrm{nmol} / \mathrm{L}(n=30)$. Thus, ionomycin-treated siTRPC1 cells have a similar $\left[\mathrm{Ca}^{2+}\right]_{\mathrm{i}}$ as mock control cells. As shown in Fig. $6 \mathrm{c}$, the global increase of $\left[\mathrm{Ca}^{2+}\right]_{\mathrm{i}}$ does not influence the velocity of siTRPC1 cells, which is $0.86 \pm 0.06 \mu \mathrm{m} / \mathrm{min}$ before and $0.83 \pm 0.05 \mu \mathrm{m} / \mathrm{min}$ after the application ionomycin $(n=37)$. For comparison, mock control cells, having the same $\left[\mathrm{Ca}^{2+}\right]_{\mathrm{i}}$ as ionomycin-treated siTRPC1 cells, have a higher mean velocity $(0.93 \pm 0.05 \mu \mathrm{m} / \mathrm{min}, n=$ 24). Thus, the TRPC1-dependent locally elevated $\left[\mathrm{Ca}^{2+}\right]_{\mathrm{i}}$ at

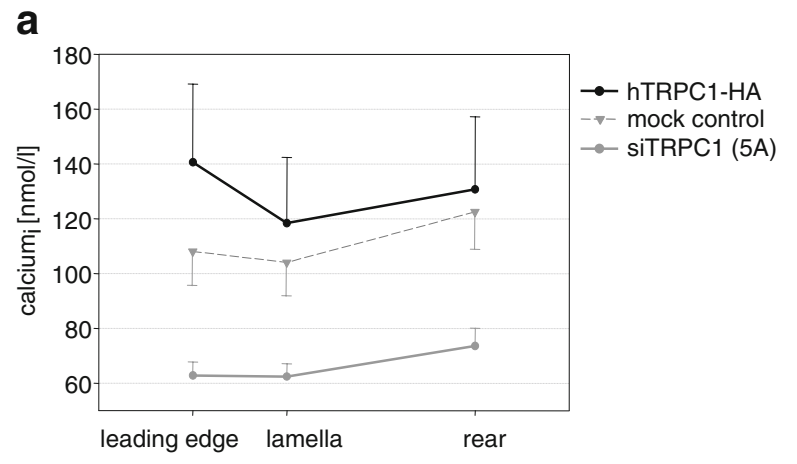

b
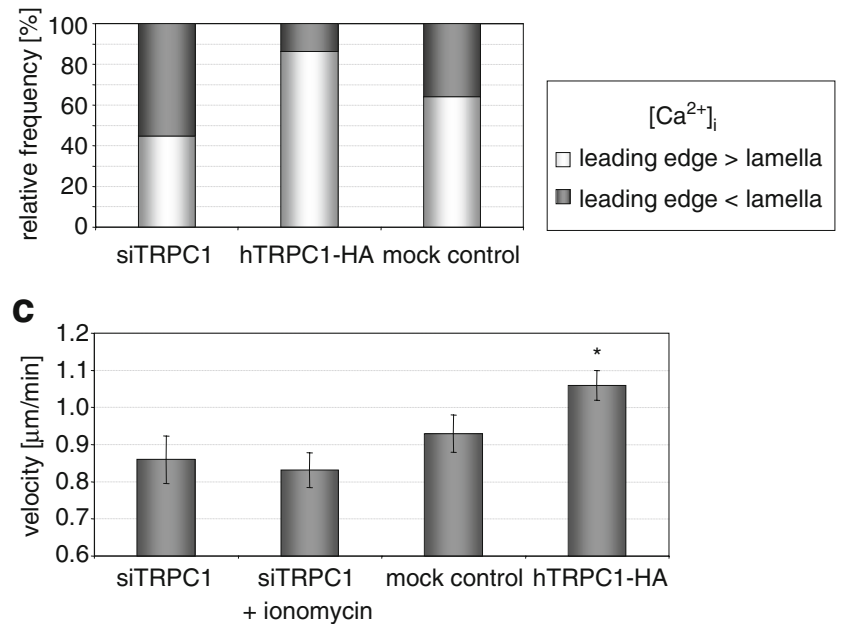

Fig. 6 TRPC1 generates a $\mathrm{Ca}^{2+}$ gradient within the lamellipodium. a $\left[\mathrm{Ca}^{2+}\right]_{\mathrm{i}}$ was detected ratiometrically with fura-2. $\left[\mathrm{Ca}^{2+}\right]_{\mathrm{i}}$ is higher at the leading edge of hTRPC1-HA (black) and mock control cells (dashed gray) than in the following lamella. Overexpression of TRPC1 leads to the formation of a steeper gradient inside the lamellipodium. In contrast, gradients of siTRPC1 cells (lower graph, gray) are only poorly developed and siTRPC1 cells have a significantly reduced resting $\left[\mathrm{Ca}^{2+}\right]_{\mathrm{i}}$. b The relative frequency of a higher local $\left[\mathrm{Ca}^{2+}\right]_{\mathrm{i}}$ in the leading edge than in the lamella (light gray) is much lower in siTRPC1 cells than in hTRPC1-HA-overexpressing cells and mock control cells. c Applying $50 \mathrm{nmol} / \mathrm{L}$ ionomycin increases $\left[\mathrm{Ca}^{2+}\right]_{\mathrm{i}}$ in siTRPC1 cells, so that the mean $\mathrm{Ca}^{2+}$ level of ionomycin-treated siTRPC1 cells is comparable to that of mock controls. However, ionomycin has no impact on the velocity of siTRPC1 cells. This indicates that the missing $\mathrm{Ca}^{2+}$ gradient within the lamellipodium of siTRPC1 cells but not lower global $\left[\mathrm{Ca}^{2+}\right]_{\mathrm{i}}$ leads to the defects in cell migration. Asterisk, $p<0.05$
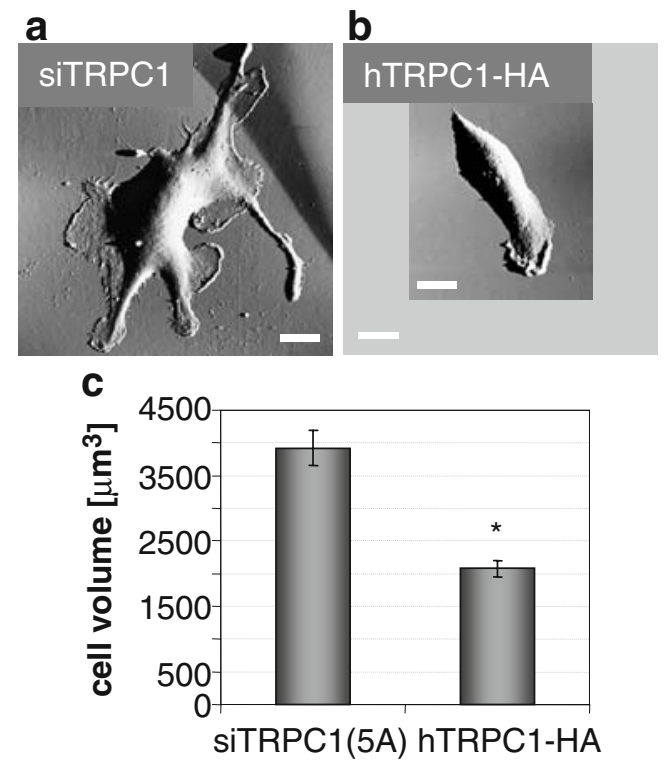

Fig. 7 Ablation of TRPC1 strongly increases the cell volume. AFM measurements reveal the vast difference in cell volume and morphology of cells with variations in TRPC1 expression. a AFM image of a fixed siTRPC1 cell (shown in the same magnification as b) that is clearly enlarged and protrudes several flat lamellipodia (scale bar in a and b $20 \mu \mathrm{m}$ ). b AFM image showing that a fixed cell with TRPC1 overexpression protrudes one prominent lamellipodium as wild-type cells. c Summary of volume measurements with AFM. The mean cell volume of siTRPC1 cells has nearly doubled $(+82 \%)$ when compared to that of hTRPC1-HA-overexpressing cells. Asterisk, $p<0.05$

the front of migrating cells appears to play an important role in coordinating migration of MDCK-F cells.

Silencing of TRPC1 expression leads to a remarkable phenotype in MDCK-F cells

Employing AFM, we could show the drastic effects of TRPC1 ablation on the morphology of the cells as displayed in Fig. 7a,b. siTRPC1 cells protrude multiple very flat lamellipodia; thus, they have a polarization defect. Moreover, the AFM technique was used to quantify the change in the cell volume. As shown in Fig. 7c, cells with hTRPC1-HA overexpression have a mean cell volume of $1,971 \pm 353 \mu \mathrm{m}^{3}$ $(n=20)$, whereas the mean cell volume of siTRPC1 cells has nearly doubled $(+82 \%)$ to $3,577 \pm 601 \mu \mathrm{m}^{3}(n=22)$.

The morphology together with the inefficient migration of TRPC1 knockdown cells prompted us to investigate whether strengthening of contacts to the substratum might be the reason for our findings. We estimated the projected surface area which corresponds to the area contacting the extracellular matrix. The mean cell area of migrating siTRPC1 cells has increased more than twofold $(3,191 \pm$ $422 \mu \mathrm{m}^{2}, n=33$ ) compared to that of TRPC1-HAoverexpressing cells and mock control cells $(1,491 \pm$ $153 \mu \mathrm{m}^{2}, n=26$ and $1,070 \pm 136 \mu \mathrm{m}^{2}, \mathrm{n}=24$, respectively; Fig. 8). Some of siTRPC1 cells even shows a fivefold rise 


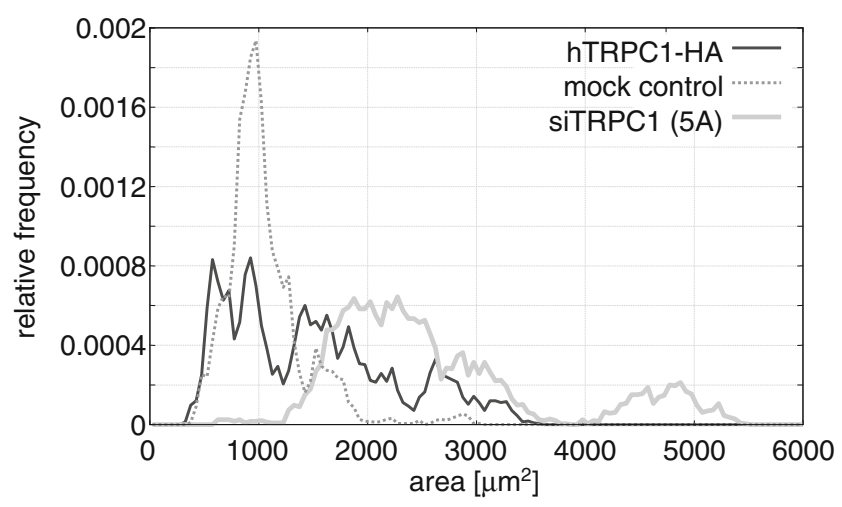

Fig. 8 Ablation of TRPC1 increases the projected cell area of migrating cells. The relative frequency of the cellular area of migrating siTRPC1 (gray) versus hTRPC1-HA-overexpressing (black) and mock control cells (dotted gray) show that knockdown of TRPC1 causes a twofold increase in the cell area. A part of the siTRPC1 cells even show a fivefold rise in the projected cell area

in the projected cell area. The increase of cell area depends on the degree of TRPC1 knockdown. When TRPC1 expression is suppressed only by $30 \%$, the average cell area is only moderately elevated $\left(1,560 \pm 129 \mu \mathrm{m}^{2}, n=26\right)$. We then challenged the adhesion strength of the cells in an adhesion assay. For this purpose, we allowed MDCK-F cells to attach to fibronectin-coated surfaces for $30 \mathrm{~min}$. Although having an increased cell area, siTRPC1 cells are surprisingly less adherent to the substratum after $30 \mathrm{~min}$ than mock controls $(n=130 \pm 16$ and $190 \pm 35$, respectively) as shown in Fig. 9. In contrast, hTRPC1-HA overexpression does not affect adhesion $(n=176 \pm 48)$. We can conclude that the increased cell area is not caused by stronger adhesion to the extracellular matrix.

\section{Discussion}

It has been shown that mechanosensitive $\mathrm{Ca}^{2+}$ channels are involved in the coordination of the protrusion of the lamellipodium with the retraction of the rear part of migrating cells $[8,9]$. But so far, the molecular identity of such channels is not known. The present study aims to connect the function of the putative stretch-activated $\mathrm{Ca}^{2+}$ channel TRPC1 to cell migration. We are well aware of the fact that the mechanosensitivity of TRPC1 is discussed controversially at present and that especially the activity of heterologously overexpressed TRPC1 in mammalian cells is problematic [14]. The spider venom GsMTx-4 is a blocker of mechanosensitive cation channels like TRPC6 $[3,40]$ and was used in this study to determine the impact of such channels on the displacement of TRPC1 knockdown and hTRPC1-HA-overexpressing cells. GsMTx-4 is three times more effective in cells overexpressing TRPC1HA than in cells with reduced expression. Moreover, it reduces $\left[\mathrm{Ca}^{2+}\right]_{\mathrm{i}}$ of hTRPC1-HA-overexpressing cells while it does not in siTRPC1 cells. One explanation for this differential effect could be that GsMTx-4 also blocks TRPC1 as reported in a review by Bowman et al. [3]. Assuming that TRPC1 is indeed a mechanosensitive channel, our experiments with GsMTx-4 would be in line with observations made in nerve growth cones. The control of their extension was strongly dependent on mechanosensitive but not on voltage-gated $\mathrm{Ca}^{2+}$ channels [19]. Related studies showed that TRPC1 is important for path finding of outgrowing neurites $[25,42]$. Moreover, TRPC1 has been implicated in electric field detection which affects directed migration of neutrophils [21]. Thus, the fact that TRPC1 is important for directed cell migration becomes apparent in various cell types.

Our study clearly shows that TRPC1 channels determine polarity and persistence of MDCK-F cells. The underlying mechanism seems to be decreasing $\mathrm{Ca}^{2+}$ concentrations from leading edge to the following lamella due to TRPC1 activity at the front. This gradient is steeper in hTRPC1HA-overexpressing cells. Although we could not visualize a concentration of the TRPC1 channel at the leading edge of MDCK-F cells as observed in neutrophils [21], we could functionally show that TRPC1 is active at the leading edge and generates a $\mathrm{Ca}^{2+}$ gradient. This finding corresponds to the fact that only the local blockade of stretch-activated $\mathrm{Ca}^{2+}$ channels at the leading edge causes a global inhibition of traction forces and cell migration [28]. The resting $\left[\mathrm{Ca}^{2+}\right]_{\mathrm{i}}$ of siTRPC 1 cells is significantly reduced, but an artificial increase in global $\left[\mathrm{Ca}^{2+}\right]_{\mathrm{i}}$ could not restore normal motility. Therefore, we propose that the missing $\mathrm{Ca}^{2+}$ gradients in siTRPC1 cells contribute to the impaired migration. The defect in cell migration is accompanied by a nonpolarized cell morphology with several very flat protrusions often coexisting at opposing poles of the cell. This suggests that TRPC1-mediated local $\mathrm{Ca}^{2+}$ signals might convey polarity and might regulate the formation of protrusions. F-actin at the front of migrating cells can be divided into two distinct

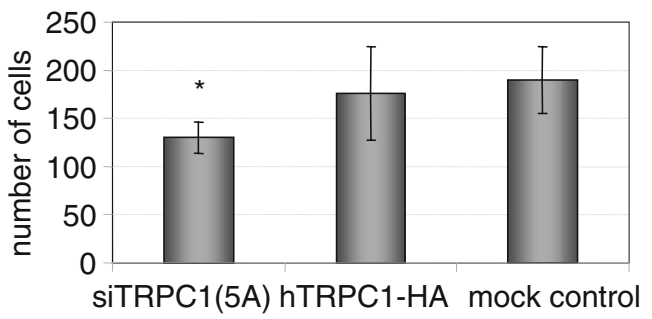

Fig. 9 siTRPC1 cells show reduced adhesion. Cells were plated on fibronectin-coated surfaces for $30 \mathrm{~min}$ at $37^{\circ} \mathrm{C}$ to allow spreading. Weakly adherent cells were washed off and adherent cells were counted. Silencing of TRPC1 expression leads to a significant reduction of the number of adherent cells $30 \mathrm{~min}$ after seeding. In contrast, there is no difference between mock controls and hTRPC1HA-expressing cells, which adhere normally. Asterisk, $p<0.05$ 
networks with characteristic dynamic and molecular properties: the lamellipodium and the following lamella [31]. The difference between the two is determined by the activity of actin-binding proteins like the F-actin severing and depolymerizing protein cofilin [6]. Active cofilin increases the number of free barbed ends of F-actin so that actin polymerization is locally enhanced [2]. The possible link to $\mathrm{Ca}^{2+}$ gradients is the fact that cofilin can be activated indirectly by elevations of $\left[\mathrm{Ca}^{2+}\right]_{\mathrm{i}}[43]$.

The productive protrusion of the lamellipodium requires the advancement of the lamella, where contractile forces are coupled to substrate adhesion [31]. In this context, it is notable that siTRPC1 cells do not only have defects in cell migration but also exhibit reduced substrate adhesion. Thus, balanced adhesion turnover seems to be perturbed in siTRPC1 cells due to the missing determination of leading edge and lamella. In general, the coordination of traction forces along the axis of movement is regulated by $\mathrm{Ca}^{2+}$ influx through $\mathrm{Ca}^{2+}$ channels. Traction forces are maintained via adhesion contacts until retraction occurs [9]. This is in line with observations made in fibroblasts. Gadolinium, an unspecific inhibitor of stretch-activated cation channels, inhibited $\mathrm{Ca}^{2+}$ entry and decreases the phosphorylation at focal adhesions in migrating fibroblasts [28]. Thus, membrane stretch induces the generation of traction forces by $\mathrm{Ca}^{2+}$ influx together with tyrosine phosphorylation of paxillin at focal adhesions and subsequent accumulation of cortical actin [13]. Silencing of TRPC1 might disturb the coordinated responses of leading edge and lamella to mechanical input. This might be the reason for the observed "noisy," inefficient movement with a strong decrease of the mean squared displacement.

The prominent effect of TRPC1 ablation on the cell morphology, a large increase of the projected cell area, and a doubling of the cell volume could be a reflection of impaired mechanosensation at the level of the plasma membrane. An artificial decrease of membrane tension over the entire cell membrane is directly correlated with the formation of multiple lamellipodial extensions pointing to an inverse relation between membrane tension and extension [33]. Thus, it will be one of the challenges for future studies to directly link cell migration to the putative mechanosensory function of TRPC1 or to that of other mechanosensitive $\mathrm{Ca}^{2+}$ channels in cell migration.

Acknowledgments The authors thank Michael Zhu from the Ohio State University for kindly providing us with a plasmid of the human HA-tagged TRPC1. This work was supported by a grant from the Rolf-Dierichs-Stiftung of the Medical Faculty of the University of Münster (grant number 193423) to A.F., by the IZKF Münster (grant number Schw2/030/08) and by the Deutsche Forschungsgemeinschaft (grant number Schw 407/9-3, 10-1) to A.S. B.N. was supported by a Human Frontiers Research Grant (RGP 32/2004), the Excellentiefinanciering (Flanders, EF/95/010), and the Interuniversity Poles of Attraction Program, Prime Minister's Office IUAP.

\section{References}

1. Arora PD, McCulloch CA (1996) Dependence of fibroblast migration on actin severing activity of gelsolin. J Biol Chem 271:20516-20523

2. Bamburg JR (1999) Proteins of the ADF/cofilin family: essential regulators of actin dynamics. Annu Rev Cell Dev Biol 15:185-230

3. Bowman CL, Gottlieb PA, Suchyna TM, Murphy YK, Sachs F (2007) Mechanosensitive ion channels and the peptide inhibitor GsMTx-4: history, properties, mechanisms and pharmacology. Toxicon 49:249-270

4. Brummelkamp TR, Bernards R, Agami R (2002) A system for stable expression of short interfering RNAs in mammalian cells. Science 296:550-553

5. Brundage RA, Fogarty KE, Tuft RA, Fay FS (1993) Chemotaxis of newt eosinophils: calcium regulation of chemotactic response. Am J Physiol 265:C1527-C1543

6. Delorme V, Machacek M, DerMardirossian C, Anderson KL, Wittmann T, Hanein D, Waterman-Storer C, Danuser G, Bokoch GM (2007) Cofilin activity downstream of Pak1 regulates cell protrusion efficiency by organizing lamellipodium and lamella actin networks. Dev Cell 13:646-662

7. Dieterich P, Klages R, Preuss R, Schwab A (2008) Anomalous dynamics of cell migration. Proc Natl Acad Sci U S A 105:459-463

8. Doyle AD, Lee J (2005) Cyclic changes in keratocyte speed and traction stress arise from $\mathrm{Ca} 2+$-dependent regulation of cell adhesiveness. J Cell Sci 118:369-379

9. Doyle A, Marganski W, Lee J (2004) Calcium transients induce spatially coordinated increases in traction force during the movement of fish keratocytes. J Cell Sci 117:2203-2214

10. Dreval V, Dieterich P, Stock C, Schwab A (2005) The role of Ca2+ transport across the plasma membrane for cell migration. Cell Physiol Biochem 16:119-126

11. Eddy RJ, Pierini LM, Matsumura F, Maxfield FR (2000) Ca2+dependent myosin II activation is required for uropod retraction during neutrophil migration. J Cell Sci 113(Pt 7):1287-1298

12. Franco SJ, Huttenlocher A (2005) Regulating cell migration: calpains make the cut. J Cell Sci 118:3829-3838

13. Glogauer M, Arora P, Yao G, Sokholov I, Ferrier J, McCulloch CA (1997) Calcium ions and tyrosine phosphorylation interact coordinately with actin to regulate cytoprotective responses to stretching. J Cell Sci 110(Pt 1):11-21

14. Gottlieb P, Folgering J, Maroto R, Raso A, Wood TG, Kurosky A, Bowman C, Bichet D, Patel A, Sachs F, Martinac B, Hamill OP, Honore E (2008) Revisiting TRPC1 and TRPC6 mechanosensitivity. Pflugers Arch 455(6):1097-1103

15. Grynkiewicz G, Poenie M, Tsien RY (1985) A new generation of $\mathrm{Ca} 2+$ indicators with greatly improved fluorescence properties. J Biol Chem 260:3440-3450

16. Hahn K, DeBiasio R, Taylor DL (1992) Patterns of elevated free calcium and calmodulin activation in living cells. Nature 359:736-738

17. Hamill OP, Martinac B (2001) Molecular basis of mechanotransduction in living cells. Physiol Rev 81:685-740

18. Huang JB, Kindzelskii AL, Clark AJ, Petty HR (2004) Identification of channels promoting calcium spikes and waves in HT1080 tumor cells: their apparent roles in cell motility and invasion. Cancer Res 64:2482-2489

19. Jacques-Fricke BT, Seow Y, Gottlieb PA, Sachs F, Gomez TM (2006) $\mathrm{Ca} 2+$ influx through mechanosensitive channels inhibits neurite outgrowth in opposition to other influx pathways and release from intracellular stores. J Neurosci 26:5656-5664

20. Kimura C, Oike M, Koyama T, Ito Y (2001) Alterations of Ca2+ mobilizing properties in migrating endothelial cells. Am J Physiol Heart Circ Physiol 281:H745-H754 
21. Kindzelskii AL, Petty HR (2005) Ion channel clustering enhances weak electric field detection by neutrophils: apparent roles of SKF96365-sensitive cation channels and myeloperoxidase trafficking in cellular responses. Eur Biophys J 35:1-26

22. Komuro H, Rakic P (1996) Intracellular Ca2+ fluctuations modulate the rate of neuronal migration. Neuron 17:275-285

23. Lawson MA, Maxfield FR (1995) $\mathrm{Ca}(2+)$ - and calcineurindependent recycling of an integrin to the front of migrating neutrophils. Nature 377:75-79

24. Lee J, Ishihara A, Oxford G, Johnson B, Jacobson K (1999) Regulation of cell movement is mediated by stretch-activated calcium channels. Nature 400:382-386

25. Li Y, Jia YC, Cui K, Li N, Zheng ZY, Wang YZ, Yuan XB (2005) Essential role of TRPC channels in the guidance of nerve growth cones by brain-derived neurotrophic factor. Nature 434:894-898

26. Mandeville JT, Ghosh RN, Maxfield FR (1995) Intracellular calcium levels correlate with speed and persistent forward motion in migrating neutrophils. Biophys J 68:1207-1217

27. Maroto R, Raso A, Wood TG, Kurosky A, Martinac B, Hamill OP (2005) TRPC1 forms the stretch-activated cation channel in vertebrate cells. Nat Cell Biol 7:179-185

28. Munevar S, Wang YL, Dembo M (2004) Regulation of mechanical interactions between fibroblasts and the substratum by stretchactivated Ca2+ entry. J Cell Sci 117:85-92

29. Oberleithner H, Westphale HJ, Gassner B (1991) Alkaline stress transforms Madin-Darby canine kidney cells. Pflugers Arch 419:418-420

30. Pedersen SF, Nilius B (2007) Transient receptor potential channels in mechanosensing and cell volume regulation. Methods Enzymol 428:183-207

31. Ponti A, Machacek M, Gupton SL, Waterman-Storer CM, Danuser G (2004) Two distinct actin networks drive the protrusion of migrating cells. Science 305:1782-1786

32. Rao JN, Platoshyn O, Golovina VA, Liu L, Zou T, Marasa BS, Turner DJ, Yuan JX, Wang JY (2006) TRPC1 functions as a storeoperated $\mathrm{Ca} 2+$ channel in intestinal epithelial cells and regulates early mucosal restitution after wounding. Am J Physiol Gastrointest Liver Physiol 290:G782-G792

33. Raucher D, Sheetz MP (2000) Cell spreading and lamellipodial extension rate is regulated by membrane tension. J Cell Biol 148:127-136

34. Ridley AJ, Schwartz MA, Burridge K, Firtel RA, Ginsberg MH, Borisy G, Parsons JT, Horwitz AR (2003) Cell migration: integrating signals from front to back. Science 302:17041709

35. Schneider SW, Pagel P, Rotsch C, Danker T, Oberleithner H, Radmacher M, Schwab A (2000) Volume dynamics in migrating epithelial cells measured with atomic force microscopy. Pflugers Arch 439:297-303

36. Schwab A, Finsterwalder F, Kersting U, Danker T, Oberleithner H (1997) Intracellular Ca2+ distribution in migrating transformed epithelial cells. Pflugers Arch 434:70-76

37. Schwab A, Rossmann H, Klein M, Dieterich P, Gassner B, Neff C, Stock C, Seidler U (2005) Functional role of Na+-HCO3cotransport in migration of transformed renal epithelial cells. J Physiol 568(Pt 2):445-458

38. Schwab A, Nechyporuk-Zloy V, Fabian A, Stock C (2007) Cells move when ions and water flow. Pflugers Arch 453:421-432

39. Sharif-Naeini R, Dedman A, Folgering JH, Duprat F, Patel A, Nilius B, Honore E (2008) TRP channels and mechanosensory transduction: insights into the arterial myogenic response. Pflugers Arch DOI 10.1007/s00424-007-0432-y

40. Spassova MA, Hewavitharana T, Xu W, Soboloff J, Gill DL (2006) A common mechanism underlies stretch activation and receptor activation of TRPC6 channels. Proc Natl Acad Sci U S A 103:16586-16591

41. Suchyna TM, Johnson JH, Hamer K, Leykam JF, Gage DA, Clemo HF, Baumgarten CM, Sachs F (2000) Identification of a peptide toxin from Grammostola spatulata spider venom that blocks cationselective stretch-activated channels. J Gen Physiol 115:583-598

42. Wang GX, Poo MM (2005) Requirement of TRPC channels in netrin-1-induced chemotropic turning of nerve growth cones. Nature 434:898-904

43. Wang Y, Shibasaki F, Mizuno K (2005) Calcium signal-induced cofilin dephosphorylation is mediated by slingshot via calcineurin. J Biol Chem 280:12683-12689

44. Waning J, Vriens J, Owsianik G, Stuwe L, Mally S, Fabian A, Frippiat C, Nilius B, Schwab A (2007) A novel function of capsaicin-sensitive TRPV1 channels: involvement in cell migration. Cell Calcium 42:17-25

45. Wes PD, Chevesich J, Jeromin A, Rosenberg C, Stetten G, Montell C (1995) TRPC1, a human homolog of a Drosophila store-operated channel. Proc Natl Acad Sci U S A 92:9652-9656

46. Zhu X, Chu PB, Peyton M, Birnbaumer L (1995) Molecular cloning of a widely expressed human homologue for the Drosophila trp gene. FEBS Lett 373:193-198 